\title{
An Automated Method for Characterizing the Relaxedness of Galaxy Clusters
}

\author{
MATT GEORGE \\ HARVARD COLLEGE \\ OfFICE OF SCIENCE, SCIENCE UNDERGRAdUATE LABORATORY INTERNSHIP \\ STANFORd LineAR ACCELERATOR CENTER \\ MENLO PARK, CA 94025
}

August 19, 2005

Prepared in partial fulfillment of the requirements of the Office of Science, Department of Energy Science Undergraduate Laboratory Internship under the direction of Dr. Steve Allen and Dr. Greg Madejski in the Kavli Institute for Particle Astrophysics and Cosmology at the Stanford Linear Accelerator Center.

Participant:

Signature

Advisors:

Signature

Signature

Work supported by Department of Energy contract DE-AC02-76SF00515 


\section{Contents}

$\begin{array}{ll}\text { Abstract } & 3\end{array}$

1 Introduction $\quad 4$

2 Procedures $\quad 5$

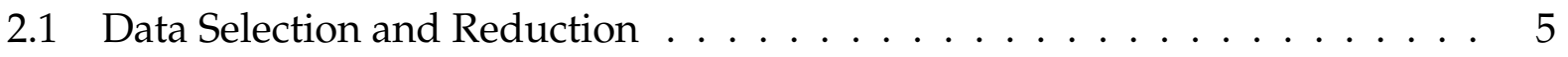

2.2 Elliptical Isophote Fitting . . . . . . . . . . . . . . . . . 6

2.3 Power Ratios . . . . . . . . . . . . . . . . . . . . . 7

2.4 Other Tests . . . . . . . . . . . . . . . . . . 8

3 Analysis $\quad 9$

4 Conclusion $\quad 10$

5 Acknowledgements $\quad 11$

$\begin{array}{ll}\text { References } & 11\end{array}$

\section{List of Tables}

1 Information about the cluster sample: cluster name, Chandra observation ID, instrument (ACIS-S or ACIS-I), exposure length, redshift, and training group (Relaxed, Unrelaxed, or none). . . . . . . . . . . . . . 14

\section{List of Figures}

1 Elliptical isophotes fit to A2029, a relaxed cluster (left), and A520, an unrelaxed cluster (right) . . . . . . . . . . . . . . . . . . . . . . . . . . . . 15

2 A King model fit to the surface brightness profile of A478 . . . . . . . . . 16

3 A plot of power ratios demonstrating the correlation of the decreasing relaxedness of the clusters from bottom left to top right with the increasing values of their power ratios $P_{3} / P_{0}$ and $P_{2} / P_{0}$. Figure from [9]. . . . . . . . 16

4 Index parameter, $\beta$, plotted against core radius from the King model fits to the catalog. Relaxed clusters exhibit smaller values of $r_{c}$ than unrelaxed clusters. . . . . . . . . . . . . . . . . . . 17

5 Centroid shifts for the catalog. The relaxed group shows smaller shifts than the group of unrelaxed clusters. . . . . . . . . . . . . . . . 17 


\title{
An Automated Method for Characterizing the Relaxedness of Galaxy Clusters
}

\author{
M.R. George ${ }^{1}$, S.W. Allen ${ }^{2}$, G.M. Madejski² \\ 1. Harvard College, Cambridge, MA 02138, USA. \\ 2. Kavli Institute for Particle Astrophysics and Cosmology, Stanford University, Menlo Park, CA 94025, USA.
}

August 19, 2005

\begin{abstract}
Relaxed galaxy clusters are useful tools for probing cosmological parameters like the gas mass fraction of the universe. Selecting relaxed clusters for this purpose can be a time-consuming and subjective task, so we present methods to automate parts of the process. We fit elliptical isophotes to a diverse sample of Chandra cluster data and summarize other methods for quantifying relaxedness which will be included in future work. Analysis of the results of tests from isophote fitting, combined with numerical simulations of cluster structures and comparison to previous classifications will allow us to formulate criteria for selection of relaxed clusters. We find that they tend to have core radii less than approximately $60 \mathrm{kpc}$ from King model fits, shifts in isophote centroids of less than $25 \mathrm{kpc}$ over a range in semi-major axes of several hundred $\mathrm{kpc}$, and significantly greater surface brightness profile gradients within $30 \mathrm{kpc}$ of their cores than unrelaxed clusters. These criteria will help with future cosmological work as larger amounts of cluster data are taken and need objective classification.
\end{abstract}




\section{Introduction}

Galaxy clusters are important objects for constraining cosmological parameters. Due to their immense size, they are thought to provide a representative sampling of the matter distribution of the universe. The structure within clusters can offer information about evolution over large timescales as well as the dynamics of galaxy interactions. In order to make use of the complex and diverse array of clusters, it is important to have the ability to classify them objectively.

For cosmological purposes, we often wish to select dynamically relaxed clusters for closer study. These objects can be used as a type of standard tool for cosmological mass measurements in the way that Type Ia supernovae have been used as homogeneous candles to study cosmic acceleration. Eliminating clusters with significant structure or activity allows a more careful measurement of the X-ray gas mass fraction, which offers a useful parameter in calculating the densities of matter and dark energy in the universe [2]. Unrelaxed clusters can be physically identified from their spectra if their cores have cooling times greater than the age of the universe, while the cores of relaxed clusters cool on shorter timescales [4]. Relaxed clusters are visually distinguished by their smooth spherical or ellipsoidal gas distributions and relatively sharp central peaks, and they can often be identified optically from a single large galaxy lying at the center of the gravitational well. Unrelaxed clusters may show signs of multiple peaks, asymmetric gas distributions, or merger activity between galaxies.

To date, most classification of clusters has been done by eye. [10] uses a common approach to cluster morphology by analyzing contour lines for multiple brightness peaks, asymmetries, and other irregularities to identify structure. In an effort to reduce the subjectivity introduced by human judgements, [5] presents an attempt to quantify morphologies by measuring the power ratios of polar moments in the x-ray flux distribution. This

method has recently been used in a study [4] of the cooling cores of clusters. Another approach involves fitting elliptical isophotes to images of the clusters as originally detailed 
for galaxy profiles by [8]. The shifts in centroids and position angles of the ellipses, as well as their ellipticities, offer quantitative details about the shape of the profile which can standardize judgments made by eye. More approaches are presented by [12], which measures deviations from a spherically symmetric profile with $\beta$, Lee, and Fourier elongation tests. In this paper, we present an attempt to synthesize some of these techniques with a goal of creating a single automated system for identification of relaxed galaxy clusters.

In the following section, we will outline the tests to be used in our analysis beginning with simpler ones like surface brightness profile fitting from elliptical isophotes. Methods to be implemented in the future like power ratios, as well as more complex techniques such as subtracting a model cluster profile from the data with Markov Chain Monte Carlo analysis will be discussed toward the end of $\S 2$. The results of the tests we have performed will be summarized in $\S 3$. In $\S 4$, we will summarize our findings and discuss future uses for the tools created, including the prospect of creating a unified and efficiently computed index to characterize the relaxedness of a cluster.

\section{Procedures}

\subsection{Data Selection and Reduction}

In order to determine the shapes and evolutionary states of galaxy clusters, we can view the intracluster gas, which has been heated to temperatures sufficiently high to emit $x-$ rays. We use data freely available from the Chandra archive, with clusters in the redshift range $0.06<\mathrm{z}<0.45$. We use Chandra data for its high spatial resolution, which is significantly greater than prior instruments. Clusters were selected from those observed by [1], as well as those listed in the ROSAT Brightest Cluster Sample and its extension, produced by [6, 7] and the REFLEX catalog introduced by [3]. The clusters used in this paper are presented along with their redshifts and other relevant information in Table 1. 
With the intention of creating a fast method for selecting relaxed clusters from larger samples or surveys, we wished to minimize the amount of data reduction needed. We chose to use images from the archive's standard processing pipeline which had already been filtered for bad pixels and only contained the two-dimensional spatial information, rather than including the spectral data and reducing the images more thoroughly. Images taken by ACIS-S were preferred over those from ACIS-I due to gaps in the chip layout, but masking will be performed along with the power ratio analysis in order to account for these flaws and to allow for the inclusion of images from both instruments. Additionally, bright sources not belonging to the cluster and regions of high background will be removed using the reduction and power ratio code from [4]. For certain analyses, AGN or other bright sources like central dominant galaxies must be masked from the centers of some clusters.

\subsection{Elliptical Isophote Fitting}

A simple way to extract useful information about the shape of a cluster is to fit elliptical isophotes to images of the profile. We used the ellipse function in IRAF to carry out this task, allowing the position angle, ellipticity, and centroid position to vary while fitting ellipses at fixed semi-major axes (SMA) separated in increments of one pixel. Examples of elliptical fits superposed on the Chandra images of a relaxed and an unrelaxed cluster are shown in Figure 1.

This process is not easily automated, since fitting ellipses to a diverse array of clusters requires attention to be given to the program's parameters. For instance, clusters with significant substructure need to have initial ellipses fit at larger distances from the centroid in order to include the whole profile, while more relaxed clusters have smaller core radii and must initially be fit much closer to the center. These differences themselves could provide useful information about the relaxedness of a cluster, but we prefer a more rigorous method than relying on the peculiarities of a fitting program to classify clusters. 
Once the information is obtained from the ellipse fitting process, we perform statistical analyses to differentiate between clusters. Using IDL code, we fit a King model of the form $S(r)=S_{0}\left(1+\left(\frac{r}{r_{c}}\right)^{2}\right)^{-3 \beta+\frac{1}{2}}$ to the surface brightness profile, where $S$ is the average number of photon counts across the isophote at semi-major axis $r, S_{0}$ is a free normalization parameter, $r_{c}$ is the core radius in pixels, and the third free parameter is the slope index $\beta$. This model was determined to work better than IRAF's built-in galaxy profile model, a sum of two exponentials and a constant, after considering that the King model has half as many free parameters while still providing a good visual fit. A sample comparison of a King model fit to surface brightness profile data is shown in Figure 2.

A related measurement for determining the sharpness of the peak of the surface brightness profile is its gradient. Since relaxed clusters can generally be expected to have strongly centralized luminosity profiles, the magnitudes of their gradients should be greater than those for unrelaxed clusters.

Another useful test for relaxedness that is readily available from the isophote data comes from measuring the distances that the ellipse centroids shift with SMA. Similarly, the changes in relative position angles of the ellipses can also provide information about the structure of a cluster. A relaxed cluster is expected to have a smooth and symmetric spatial distribution of x-ray emitting gas, which would result in concentric ellipses without much variation in position angle. Relaxed clusters can be elliptical rather than spherical because the relaxation time between these states is significantly longer than the age of the universe. Thus, while some may include tests of elongation to characterize relaxedness (e.g. [12]), we generally ignore the ellipticity in our analysis unless it is extreme or highly uncertain, which often indicates an isophote fit of poor quality.

\subsection{Power Ratios}

We intended to apply the method from [5] of multipole expansion of the two-dimensional gravitational potential to calculate power ratios for the sample. Our work with this ap- 
proach is presently incomplete, but the process will be explained here because it will be used in future work.

The power of an $n^{\text {th }}$ order polar moment is denoted as $P_{n}$, and for statistical analysis the power is normalized to become the power ratio $P_{n} / P_{0}$. More relaxed clusters, having less substructure and greater symmetry, tend to show less power in higher order poles than unrelaxed clusters. [5] and subsequent papers (e.g. [4]) have found a good correlation between the dipole and quadrupole ratios, $P_{2} / P_{0}-P_{4} / P_{0}$. Other ratios also appear to be correlated, though generally not as strongly. Furthermore, it has been discovered that the position of a cluster along these correlation lines is related to its morphological type as determined by other visual classification schemes. Relaxed clusters have low values for $P_{2} / P_{0}$ and $P_{4} / P_{0}$, indicating that low power in higher order moments does indeed help to quantify previous classification systems. Despite its success, the significant uncertainties in this method force us to include some of the previously mentioned tests to supplement it as an indicator of relaxedness. Figure 3 shows a plot of power ratios from [9] which graphically summarizes this method.

\subsection{Other Tests}

In an attempt to find a simple yet reliable quantification of relaxedness, we focused our efforts toward elliptical isophote fitting and calculating power ratios. Other methods considered for analysis include modeling the clusters with x-ray emitting blobs using Monte Carlo simulations to determine relevant statistical parameters in the manner of [11]. The ability to compare the results of the morphological tests from this paper with the more physical results from numerical simulations will be important and useful as the methods for using relaxed clusters to constrain cosmological parameters are refined. Other options for quantifying relaxedness include tests outlined by [12] which deal mostly with different analyses of the surface brightness profile, and determination of cooling times from spectral information, as performed by [4]. These procedures have not been included in 
this work, but offer further options to supplement our tests at the expense of increased complexity and computing time.

\section{Analysis}

To determine the relaxedness of a galaxy cluster, we use a combination of results from the tests outlined above. For the purpose of comparing values obtained by these tests to previous methods of classification, we identify training sets of clusters commonly accepted as relaxed and unrelaxed. The relaxed group consists of A478, A1835, A2029, and MS2137.3-2353. The clusters identified to be the most unrelaxed are A520, A2163, A2219, and A2744.

The core radius parameter of the King model fit to the surface brightness profile helps to differentiate relaxed and unrelaxed clusters. The relaxed group shows a narrow range of small values for its core radii, while the unrelaxed clusters exhibit a far broader range of higher core radii, as seen in Figure 4. Those clusters not identified as strongly relaxed or unrelaxed lie mostly in the region of the plot near the relaxed ones. As a result of this test, we can filter out clusters with $r_{c} \gtrsim 60 \mathrm{kpc}$ from the subset of relaxed clusters we wish to use to constrain cosmological parameters.

Other parameters from the isophote fits were analyzed, including the variation of centroids and position angles of the ellipses over their range of SMA. Position angles were found to vary widely for the whole catalog, especially at larger SMA where the relative background increased. Position angles and ellipticities did not appear to be independent enough across isophotes to be reliable, and are not used in this analysis. Centroid shifts, however, did show a clear distinction between the relaxed and unrelaxed training groups, with relaxed clusters having smaller centroid shifts. Figure 5 displays the centroid shift (CS) as a function of SMA for each cluster in the catalog. We also define a new measurement, the fractional centroid shift, FCS = CS / SMA. The mean FCS for a cluster profile 
is a simple statistic for comparison of clusters and a useful indicator of relaxedness, with the relaxed group exhibiting values less than $\approx 0.1$.

The gradient of the surface brightness profile provides another approach to quantify its peakedness. This statistic measures a morphological characteristic similar to that of the core radius from the King model fit. The relaxed clusters do show significantly larger gradients than the unrelaxed group, but with the current level of sophistication of our analysis, the gradient does not provide any greater distinction between relaxedness than the core radius parameter. Future work will involve a clearer quantification of the gradient and a closer comparison between the results of these two statistics to determine which is more useful and if they can both contribute to our goal of a relaxedness index.

We have not yet been able to synthesize these tests into a single scale for relaxedness. The power ratio method will hopefully provide information about this quality that is more orthogonal to the isophote tests, allowing us to more precisely eliminate the unrelaxed clusters from cosmological studies. The data reduction methods that will be performed with the power ratio analysis will also allow us to use the ACIS-I images more reliably, making available to us a significant number of other clusters from the Chandra archive.

\section{Conclusion}

We have presented several methods of quantifying the relaxedness of galaxy clusters for the purpose of selecting relaxed ones to use in constraining cosmological parameters. These tests will be run periodically on the set of cluster images available from the Chandra archive, and future work may allow the use of data from XMM-Newton or other instruments. The catalog for $f_{\text {gas }}$ measurements in [2] will be updated as new clusters are determined to be sufficiently relaxed and new data demonstrates that clusters already existing in the catalog fail to meet our specified criteria. 
Classification of galaxy clusters can now be done without much need for human intervention or subjectivity. As mentioned above, this morphological work will be incorporated with more theoretical and physical models of cluster dynamics and cooling flows in the future. Further refinement of these tests is necessary, but our results show promise for providing an objective measurement of the relaxedness of galaxy clusters. An improved understanding of how galaxy clusters form and evolve will make them better standardized tools for cosmological studies.

\section{Acknowledgements}

This work was made possible by the Office of Science, Department of Energy Science Undergraduate Laboratory Internship program. F. Bauer graciously provided the code for power ratio analysis. M. George would also like to thank his advisors, S.W. Allen and G.M. Madejski, and the program manager, James Lindesay, for their support.

\section{References}

[1] S. W. Allen and A. C. Fabian. The relationship between cooling flows and metallicity measurements for X-ray-luminous clusters. MNRAS, 297:L63-L68, July 1998.

[2] S. W. Allen, R. W. Schmidt, H. Ebeling, A. C. Fabian, and L. van Speybroeck. Constraints on dark energy from Chandra observations of the largest relaxed galaxy clusters. MNRAS, 353:457-467, September 2004.

[3] H. Böhringer, P. Schuecker, L. Guzzo, C. A. Collins, W. Voges, R. G. Cruddace, A. Ortiz-Gil, G. Chincarini, S. De Grandi, A. C. Edge, H. T. MacGillivray, D. M. Neumann, S. Schindler, and P. Shaver. The ROSAT-ESO Flux Limited X-ray (REFLEX) Galaxy cluster survey. V. The cluster catalogue. A\&A, 425:367-383, October 2004. 
[4] F. E. Bauer, A. C. Fabian, J. S. Sanders, S. W. Allen, and R. M. Johnstone. The prevalence of cooling cores in clusters of galaxies at $\mathrm{z}^{\sim}$ 0.15-0.4. MNRAS, 359:1481-1490, June 2005.

[5] D. A. Buote and J. C. Tsai. Quantifying the Morphologies and Dynamical Evolution of Galaxy Clusters. II. Application to a Sample of ROSAT Clusters. ApJ, 458:27-+, February 1996.

[6] H. Ebeling, A. C. Edge, S. W. Allen, C. S. Crawford, A. C. Fabian, and J. P. Huchra. The ROSAT Brightest Cluster Sample - IV. The extended sample. MNRAS, 318:333340, October 2000.

[7] H. Ebeling, A. C. Edge, H. Bohringer, S. W. Allen, C. S. Crawford, A. C. Fabian, W. Voges, and J. P. Huchra. The ROSAT Brightest Cluster Sample - I. The compilation of the sample and the cluster $\log N-\log S$ distribution. MNRAS, 301:881-914, December 1998.

[8] R. I. Jedrzejewski. CCD surface photometry of elliptical galaxies. I - Observations, reduction and results. MNRAS, 226:747-768, June 1987.

[9] T. E. Jeltema, C. R. Canizares, M. W. Bautz, and D. A. Buote. The Evolution of Structure in X-Ray Clusters of Galaxies. ApJ, 624:606-629, May 2005.

[10] C. Jones and W. Forman. Einstein Observatory Images of Clusters of Galaxies. ApJ, 511:65-83, January 1999.

[11] J. R. Peterson, P. J. Marshall, and K. Andersson. Smoothed Particle Inference: A KiloParametric Method for X-ray Galaxy Cluster Modeling. ArXiv Astrophysics e-prints, July 2005.

[12] P. Schuecker, H. Böhringer, T. H. Reiprich, and L. Feretti. A systematic study of X- 
ray substructure of galaxy clusters detected in the ROSAT All-Sky Survey. A\&A, 378:408-427, November 2001. 


\section{Tables}

\begin{tabular}{|c|c|c|c|c|c|}
\hline Name & ID & ACIS & Exp. (ks) & $\mathrm{z}$ & Group \\
\hline A 478 & 1669 & S & 42.9400 & 0.0880000 & R \\
\hline A 478 & 6102 & $\mathrm{I}$ & 10.1300 & 0.0880000 & $\mathrm{R}$ \\
\hline A520 & 4215 & $\mathrm{I}$ & 67.1500 & 0.199000 & $\mathrm{U}$ \\
\hline A586 & 530 & $\mathrm{I}$ & 10.1700 & 0.171000 & \\
\hline A611 & 3194 & $S$ & 36.5900 & 0.288000 & \\
\hline A963 & 903 & $S$ & 36.7600 & 0.206000 & \\
\hline A1068 & 1652 & S & 27.1700 & 0.137500 & \\
\hline A1758a & 2213 & $S$ & 59.0900 & 0.279000 & \\
\hline A1795 & 493 & $S$ & 19.8800 & 0.0624760 & \\
\hline A1835 & 495 & S & 19.7700 & 0.253200 & $\mathrm{R}$ \\
\hline A2029 & 4977 & $S$ & 78.9100 & 0.0770000 & $\mathrm{R}$ \\
\hline A2142 & 1228 & S & 12.2600 & 0.0909000 & \\
\hline A2163 & 1653 & $\mathrm{I}$ & 72.0900 & 0.203000 & $\mathrm{U}$ \\
\hline A2204 & 499 & $S$ & 10.2000 & 0.152300 & \\
\hline A2219 & 896 & S & 42.8400 & 0.228000 & $\mathrm{U}$ \\
\hline A2390 & 4193 & $S$ & 96.3200 & 0.228000 & \\
\hline A2744 & 2212 & S & 25.1400 & 0.308000 & $\mathrm{U}$ \\
\hline IRAS09104+4109 & 509 & S & 9.17000 & 0.442000 & \\
\hline MS1358.4+6245 & 516 & $S$ & 54.7600 & 0.328000 & \\
\hline MS2137.3-2353 & 928 & S & 44.1700 & 0.313000 & $\mathrm{R}$ \\
\hline PKS0745-191 & 2427 & S & 18.0900 & 0.102800 & \\
\hline RXCJ2351.6-2605 & 2214 & S & 9.77000 & 0.230000 & \\
\hline RXJ1347.5-1145 & 507 & S & 10.1200 & 0.451000 & \\
\hline RXJ1532.9+3021 & 1649 & $S$ & 9.49000 & 0.345000 & \\
\hline Z2701 & 3195 & $S$ & 27.2700 & 0.214000 & \\
\hline
\end{tabular}

Table 1: Information about the cluster sample: cluster name, Chandra observation ID, instrument (ACIS-S or ACIS-I), exposure length, redshift, and training group (Relaxed, Unrelaxed, or none). 


\section{Figures}
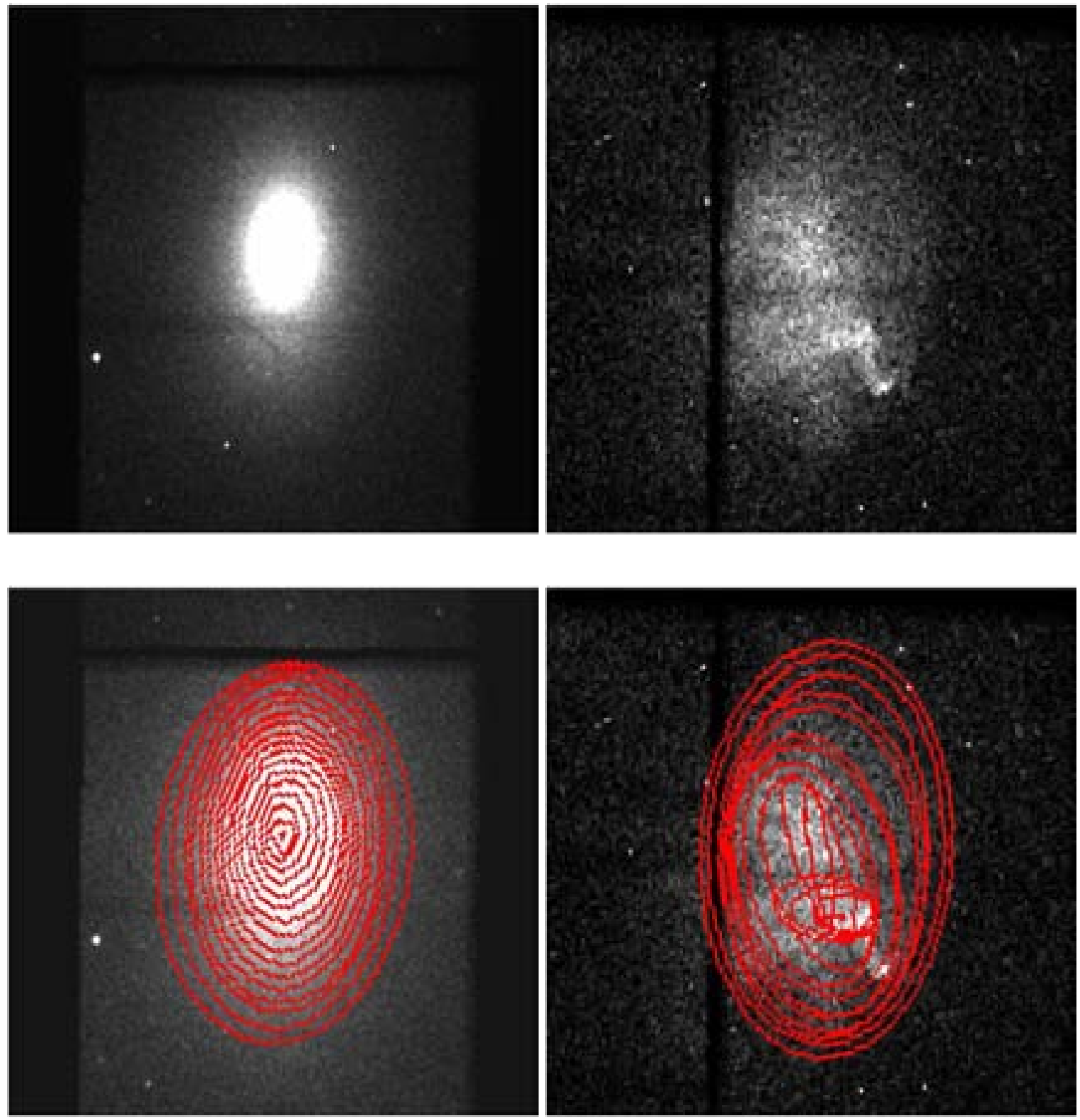

Figure 1: Elliptical isophotes fit to A2029, a relaxed cluster (left), and A520, an unrelaxed cluster (right). 


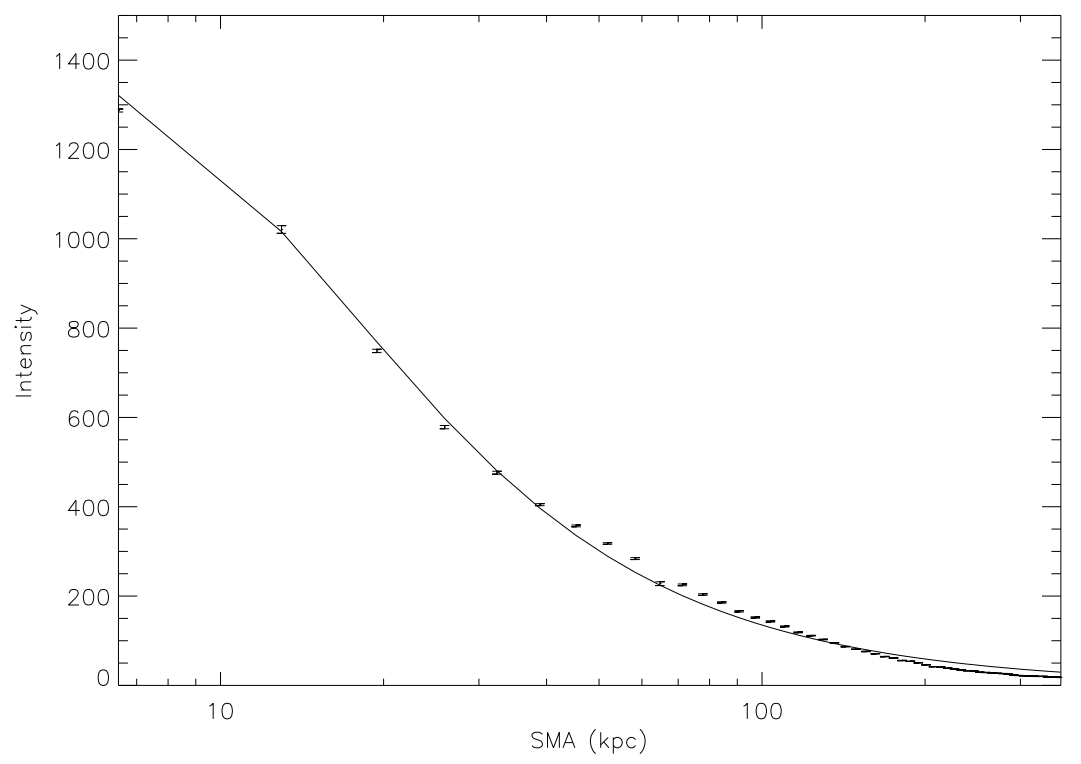

Figure 2: A King model fit to the surface brightness profile of A478

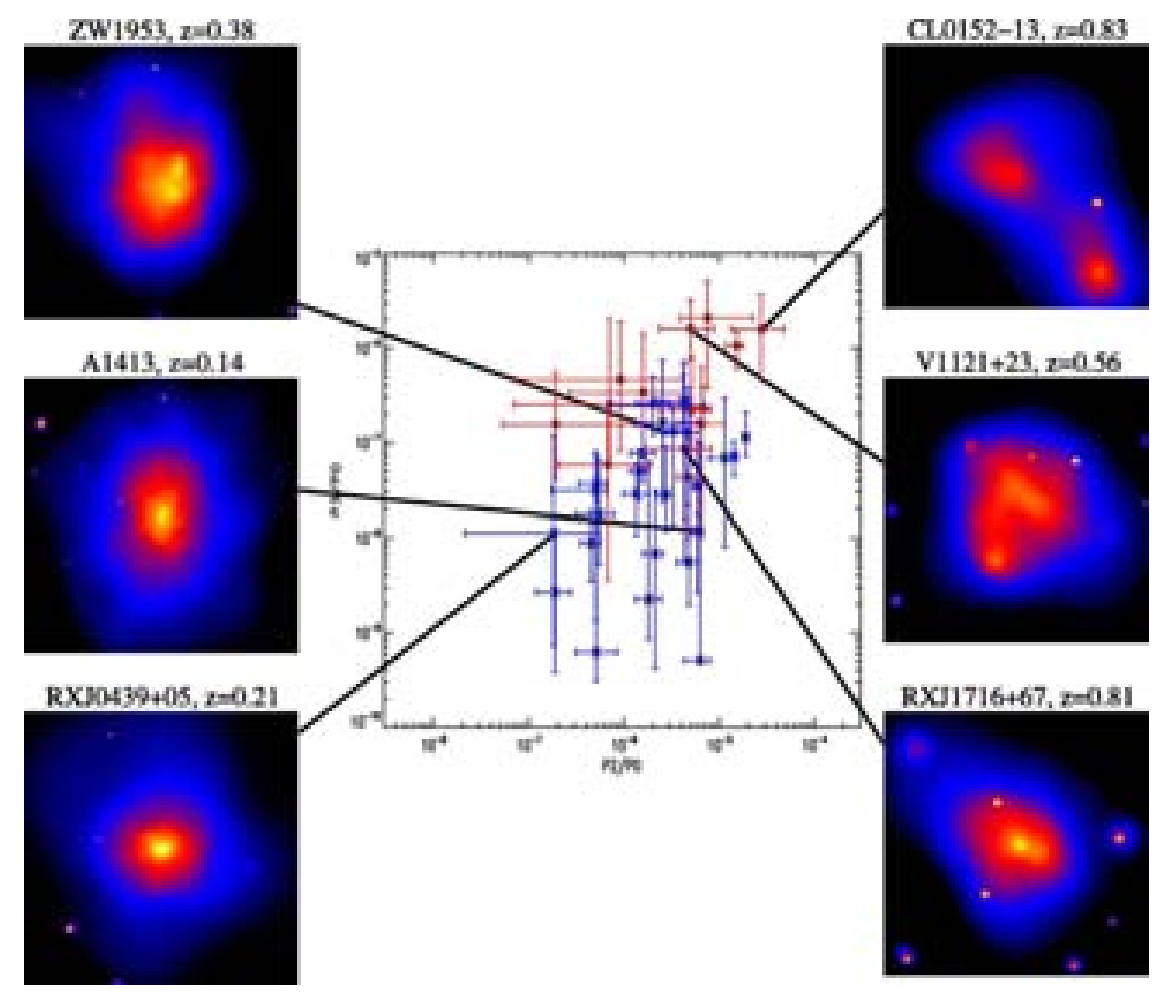

Figure 3: A plot of power ratios demonstrating the correlation of the decreasing relaxedness of the clusters from bottom left to top right with the increasing values of their power ratios $P_{3} / P_{0}$ and $P_{2} / P_{0}$. Figure from [9]. 


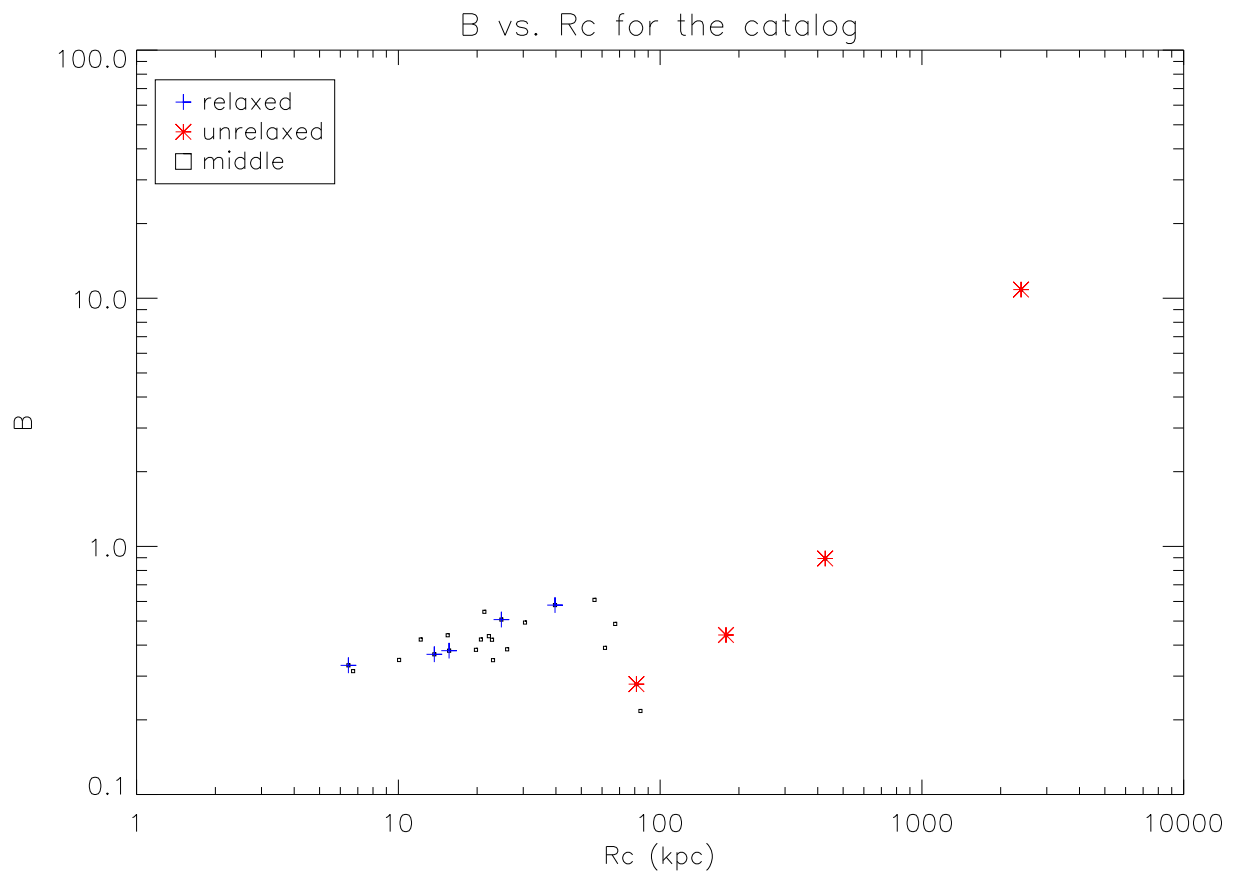

Figure 4: Index parameter, $\beta$, plotted against core radius from the King model fits to the catalog. Relaxed clusters exhibit smaller values of $r_{c}$ than unrelaxed clusters.

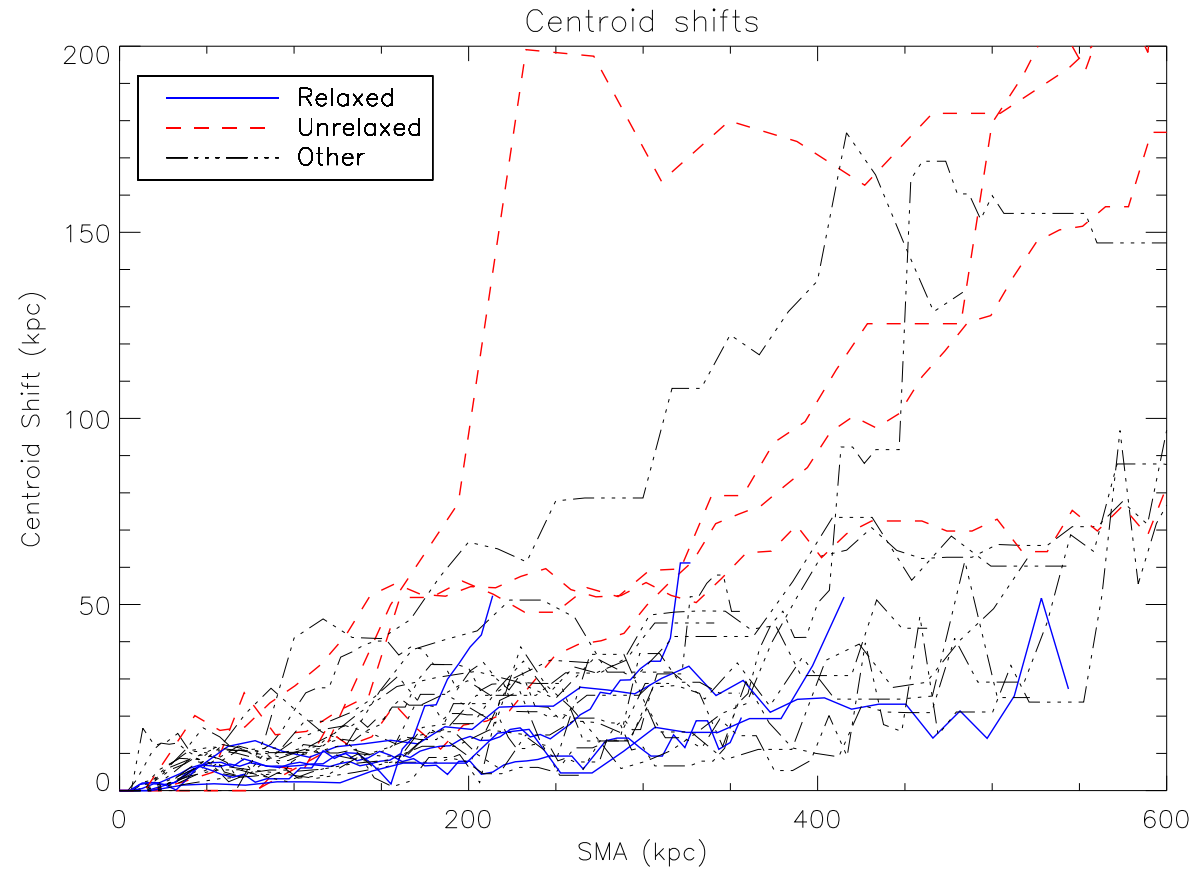

Figure 5: Centroid shifts for the catalog. The relaxed group shows smaller shifts than the group of unrelaxed clusters. 